Published in final edited form as:

J Pineal Res. 2010 September ; 49(2): 106-114. doi:10.1111/j.1600-079X.2010.00771.x.

\title{
Analysis of $\boldsymbol{N}^{1}$-acetyl- $\mathbf{N}^{2}$-formyl-5-methoxykynuramine/ $\mathbf{N}^{1}$ - acetyl-5-methoxy-kynuramine formation from melatonin in mice
}

\author{
Sida Niu, ${ }^{1, a, b}$, Feng $\mathrm{Li}^{1, \mathrm{a}}$, Dun-Xian Tan ${ }^{2}$, Lirong Zhang ${ }^{1, \mathrm{c}}$, Jeffrey R. Idle ${ }^{3}$, Frank J. \\ Gonzalez ${ }^{4}$, and Xiaochao $\mathrm{Ma}^{1}$ \\ ${ }^{1}$ Department of Pharmacology, Toxicology and Therapeutics, University of Kansas Medical \\ Center, KS, USA \\ ${ }^{2}$ Department of Cellular and Structural Biology, University of Texas Health Science Center, San \\ Antonio, TX, USA \\ ${ }^{3}$ Institute of Pharmacology, 1st Faculty of Medicine, Charles University, Praha, Czech Republic \\ ${ }^{4}$ Laboratory of Metabolism, Center for Cancer Research, National Cancer Institute, National \\ Institutes of Health, Bethesda, MD, USA
}

\begin{abstract}
The interactions of melatonin, a potent endogenous antioxidant, with reactive oxygen species generate several products that include $N^{1}$-acetyl- $N^{2}$-formyl-5-methoxykynuramine (AFMK) and $N^{1}$-acetyl-5-methoxykynuramine (AMK). The physiological or pathological significance of AFMK/AMK formation during the process of melatonin metabolism in mammals has not been clarified. Using a metabolomic approach in the current study, the AFMK/AMK pathway was thoroughly investigated both in mice and humans. Unexpectedly, AFMK and AMK were not identified in the urine of humans nor in the urine, feces or tissues (including liver, brain, and eyes) in mice under the current experimental conditions. Metabolomic analysis did identify novel metabolites of AMK, i.e. hydroxy-AMK and glucuronide-conjugated hydroxy-AMK. These two newly identified metabolites were, however, not found in the urine of humans. In addition, oxidative stress induced by acetaminophen in the mouse model did not boost AFMK/AMK formation. These data suggest that AFMK/AMK formation is not a significant pathway of melatonin disposition in mice, even under conditions of oxidative stress.
\end{abstract}

\section{Keywords}

antioxidant; melatonin; metabolism; $N^{1}$-acetyl-5-methoxykynuramine; $N^{1}$-acetyl- $N^{2}$-formyl-5methoxykynuramine

\footnotetext{
Address reprint requests to Dr. Xiaochao Ma, Department of Pharmacology, Toxicology and Therapeutics, University of Kansas Medical Center, 4089 KLSIC, MS 1018, 3901 Rainbow Boulevard, Kansas City, KS 66160, USA. xma2@ kumc.edu.

aSida Niu and Feng Li contribute equally to this study.

bPresent address: School of Engineering, University of Kansas.

${ }^{\mathrm{c}}$ Present address: Department of Pharmacology, School of Medicine, Zhengzhou University, Zhengzhou, China.
} 


\section{Introduction}

Interest in melatonin research has been increasing during the past three decades because of its beneficial effects related to human health. Melatonin is a naturally occurring substance, which is mainly secreted by the pineal gland during the dark phase of the circadian cycle in vertebrates [1]. It is classified as the chemical expression of darkness [2]. Melatonin exhibits several important biophysiological functions. These include circadian rhythm modulation, reproductive regulation in seasonal breeders, sleep promotion, and inmunoenhancement [3]. These functions are mainly melatonin membrane receptor dependent. Melatonin is also a potent endogenous free-radical scavenger and antioxidant [4-12]. Melatonin interacts with a variety of reactive oxygen and nitrogen species, including hydroxyl radical [13], singlet oxygen [14], nitric oxide [13], hydrogen peroxide [15], peroxynitrite anion [7], hypocholorous acid [16], and the superoxide anion [17]. The antioxidant properties of melatonin are considerably different from classical antioxidants such as vitamin $\mathrm{C}$ and vitamin $\mathrm{E}$. These classic antioxidants are usually electron donors and undergo redox cycling. As a consequence, classical antioxidants are in some cases prooxidant [18]. The interaction of melatonin with free radicals results in the formation of several chemically stable products [19]. Therefore, it does not undergo redox cycling and is devoid of pro-oxidant actions. $N^{1}$ acetyl- $N^{2}$-formyl-5-methoxykynuramine (AFMK) and $N^{1}$-acetyl-5-methoxy-kynuramine (AMK) are two of these products, which are believed to be generated partially from the reactions of melatonin with free radicals [20]. AFMK and AMK themselves are also potent free-radical scavengers. The antioxidant capacities of melatonin with its secondary (AFMK) and tertiary (AMK) metabolites are classified as a free-radical cascade reaction $[9,14,21]$.

AFMK and AMK were first identified by Hirata et al. [22]. They observed that AFMK and AMK are the major derivatives of melatonin in the central nervous system of rats. AFMK and AMK formation is initiated by an enzyme, indolamine 2,3-dioxynase [23]. In addition to enzymatic pathways of metabolism, AFMK can be formed when melatonin interacts with free radicals [24]. When radiolabeled melatonin was injected intracisternally and intravenously in rats, it was reported that $35 \%$ of the total radioactivity in the urine was attributed to AMK in the rats after intracisternal injection and $15 \%$ in the rats after the intravenous administration [22]. In another study, endogenously produced AFMK was detected in the retina of rats but not in serum. Intraperitoneal administration of melatonin significantly increased serum and retinal AFMK levels. Formation of AFMK from melatonin was also confirmed by in vivo microdialysis with the probe implanted into the brain lateral ventricle [25]. However, recent studies revealed that the AFMK/AMK pathway plays an insignificant role in melatonin metabolism in mice; miniscule amounts of AFMK and AMK were detected and, in some cases, completely undetected in mouse urine after oral melatonin treatment $[26,27]$.

The discrepancies on the importance of AFMK/AMK pathway in vivo raised concerns that led to the current investigation. Firstly, we examined whether AMK was further metabolized using a metabolomic approach in the mouse model. Secondly, we investigated whether AFMK/AMK distribution is tissue specific in animals. Thirdly, we evaluated whether oxidative stress would boost AFMK/AMK formation in an animal model. 


\section{Materials and methods}

\section{Chemicals and reagents}

Melatonin, acetaminophen (APAP), glutathione (GSH), and $\beta$-nicotinamide adenine dinucleotide phosphate-reduced (NADPH) were obtained from Sigma-Aldrich (St. Louis, MO, USA). AMK and AFMK were the gift from Dr. Dun-Xian Tan (University of Texas Health Science Center, San Antonio, TX, USA). Recombinant human cytochrome P450s and human liver microsomes (HLM) were purchased from XenoTech (Lenexa, KS, USA). All the solvents for liquid chromatography and mass spectrometry were of the highest grade commercially available.

\section{Animals and treatments}

All mice (C57/BL6 strain) were maintained under a standard 12-hr dark and 12-hr light cycle with water and chow provided ad libitum. Handling was in accordance with study protocols approved by the Kansas University Medical Center Animal Care and Use Committee. Melatonin $(10 \mathrm{mg} / \mathrm{kg})$ and AMK $(10 \mathrm{mg} / \mathrm{kg})$ were administered via ball-tipped gavage needles. The mice were then housed in separate metabolic cages for $18 \mathrm{hr}$ with water and chow provided ad libitum. Urine and feces were collected and stored at $-20^{\circ} \mathrm{C}$ until further analysis. An oxidative stress mouse model was produced by treatment with APAP $(100 \mathrm{mg} / \mathrm{kg}$, ip). For tissue distribution analysis, blood, eye, brain, and liver were collected at 10,30, and 60 min after melatonin treatment.

\section{Human subjects and treatment}

Two male and two female healthy subjects, aged between 30 and 45, were recruited in this study. The study protocol was approved by the Ethics Committee of Zhengzhou University and all participants provided written informed consent. None of the subjects had a history of significant medical illness and they did not consume extensive amounts of alcohol nor did they smoke. None had taken any drug for at least $2 \mathrm{wk}$ prior to and during the study. Their health status was judged on the basis of a physical examination and a blood screen, including a complete blood count and liver function test, urine analysis, and electrocardiogram performed before the study. Each subject received a single oral dose of 10 $\mathrm{mg}$ melatonin (po) with $200 \mathrm{~mL}$ water at 8:00 P.M. After melatonin treatment, 12-hr urine samples were collected. One day earlier, 12-hr urine samples were collected and used as controls. Urine was stored at $-80^{\circ} \mathrm{C}$ until further analysis.

\section{AMK metabolism in vitro}

Incubations were conducted in $1 \times$ phosphate-buffered saline (PBS, pH 7.4), containing 50 $\mu \mathrm{m}$ AMK, $0.1 \mathrm{mg}$ HLM or 2 pmol of each cDNA-expressed P450 enzyme (control, CYP1A2, CYP2A6, CYP2B6, CYP2C8, CYP2C9, CYP2C19, CYP2D6, CYP2E1, and CYP3A4) in final volume of $200 \mu \mathrm{L}$. After $5 \mathrm{~min}$ of preincubation at $37^{\circ} \mathrm{C}$, the reaction was initiated by the addition of $10 \mu \mathrm{L}$ of NADPH $(20 \mathrm{~mm}$ ) and continued for $30 \mathrm{~min}$ with shaking. The same experiment was carried out without NADPH. All reactions were terminated by adding $200 \mu \mathrm{L}$ of cold acetonitrile to the incubation mixtures. 


\section{Sample preparation}

Urinary samples were prepared by mixing $30 \mu \mathrm{L}$ of urine and $170 \mu \mathrm{L}$ of $50 \%$ acetonitrile in water and centrifuging at 15,000 RCF for $10 \mathrm{~min}$. Feces were homogenized in 50\% acetonitrile ( $1 \mathrm{mg}$ feces in $20 \mu \mathrm{L}$ of $50 \%$ actonitrile in water), followed by vortexing and centrifugation at $15,000 \mathrm{RCF}$ for $10 \mathrm{~min}$. The supernatant was transferred to a new Eppendorf vial for a second centrifugation (15,000 RCF for $10 \mathrm{~min})$. Serum samples were prepared by mixing $25 \mu \mathrm{L}$ of serum with $25 \mu \mathrm{L}$ of $\mathrm{H}_{2} \mathrm{O}$ and $50 \mu \mathrm{L}$ of acetonitrile, followed by vortexing and centrifugation at 15,000 RCF for $10 \mathrm{~min}$. Tissues (brain, liver, and eyes) were weighed and homogenized in $\mathrm{H}_{2} \mathrm{O}\left(100 \mathrm{mg} / 400 \mu \mathrm{L} \mathrm{H}_{2} \mathrm{O}\right)$. A volume of $100 \mu \mathrm{L}$ of acetonitrile was added to $100 \mu \mathrm{L}$ of tissue mixtures. After vortexing and centrifugation (10 min at 15,000 RCF), the supernatants were transferred into sample vials. The in vitro incubation mixture was terminated with $200 \mu \mathrm{L}$ of acetonitrile and vortexed for $1 \mathrm{~min}$ and centrifuged at 15,000 RCF for $10 \mathrm{~min}$. Each supernatant was transferred to an autosampler vial and $5 \mu \mathrm{L}$ was injected to a UPLC-TOFMS system (Waters, Milford, MA, USA) combining ultra-performance liquid chromatography (UPLC) and time of flight mass spectrometry (TOFMS) for metabolites analysis, as described below.

\section{CYP1A and CYP2C activity analysis}

CYP1A and CYP2C are the major enzymes that contribute to melatonin metabolism [28]. Effect of APAP treatment on hepatic CYP1A and CYP2C activities was analyzed by monitoring melatonin 6-hydroxylation and melatonin $O$-demethylation. The livers from control and APAP-treated mice were homogenized in PBS (100 mg tissue $/ 400 \mu \mathrm{L}$ PBS) at $0^{\circ} \mathrm{C}$. The enzymatic activity analysis was carried out in $150 \mu \mathrm{L}$ homogenates, containing 50 $\mu \mathrm{m}$ melatonin. The reaction was initiated by the addition of $10 \mu \mathrm{L}$ of NADPH $(20 \mathrm{~mm})$, continued for 30 min with gentle shaking, and terminated by adding $200 \mu \mathrm{L}$ of acetonitrile to the incubation mixture.

\section{UPLC-TOFMS analysis}

A $50 \mathrm{~mm} \times 2.1 \mathrm{~mm}$ (Acquity $1.7 \mu \mathrm{m}$ ) UPLC BEH C-18 column (Waters) was used for metabolite separation. The flow rate of the mobile phase was $0.3 \mathrm{~mL} / \mathrm{min}$ with a gradient ranging from 5\% to $95 \%$ aqueous acetonitrile containing $0.1 \%$ formic acid during a $10 \mathrm{~min}$ run. The TOFMS was operated in both positive and negative ionization modes with electrospray ionization. Source temperature and desolvation temperature were set at 120 and $350^{\circ} \mathrm{C}$, respectively. Nitrogen was applied as the cone gas $(10 \mathrm{~L} / \mathrm{h})$, and desolvation gas $(700 \mathrm{~L} / \mathrm{h})$ and argon as the collision gas. TOFMS was calibrated with [glu1]-fibrinopeptide solution (range $m / z 100$ to 1000) and monitored by the intermittent injection of lock mass leucine enkeophalin $\left[(\mathrm{M}+\mathrm{H})^{+}=566.2771 \mathrm{~m} / \mathrm{z}\right]$ in real time. The capillary voltage and the cone voltage were set at $3.5 \mathrm{kV}$ and $35 \mathrm{~V}$ in positive-ion mode. Total ion mass chromatograms and mass spectra were acquired by MassLynx software in centroid format. The detection limit for AMK was $<0.05$ pmol per injection.

\section{Data analysis}

Centroided and integrated mass chromatographic data were processed by MarkerLynx software (Waters) to generate a multivariate data matrix. The corresponding data matrices 
were then exported into SIMCA-P+12 (Umetrics, Kinnelon, NJ, USA) for multivariate data analysis. Principal components analysis (PCA) and orthogonal projection to latent structures-discriminant analysis (OPLS-DA) were conducted on Pareto-scaled data. All values are expressed as the means \pm S.E.M. and group differences analyzed by paired Student's $t$ test.

\section{Results}

In humans, melatonin is principally metabolized by CYP1A isozymes to 6hydroxymelatonin, which is further conjugated with sulfate and excreted in urine [29]. Metabolomic analysis in this study confirmed that sulfated 6-hydroxymelatonin (S-O-MEL) is the dominant melatonin metabolite in humans. As shown in the loadings S-plot, S-O-MEL is significantly distinguished from the other ions (Fig. 1A). MS/MS fragmentation confirmed the structure of S-O-MEL, which had a deprotonated molecular ion 80 Da higher than that of 6-hydroxymelatonin at $m / z 327$ (negative ion mode; Fig. 1B). The CYP2Cmediated $O$-demethylation of melatonin resulting in the formation of $\mathrm{N}$-acetyl-5hydroxytryptamine ( $N$-acetylserotonin) represents a minor metabolic route in human [28]. Sulfated $N$-acetylserotonin (S-NAS), which accounts for $\sim 7 \%$ relative to the S-O-MEL pathway, was detected. However, both AFMK and AMK were not detected in urine extracts in either negative-ion or positive-ion modes (Fig. 1C). The absence of AFMK and AMK in human urine raised concerns of whether AMK was further metabolized or whether AFMK, AMK, and their metabolites were localized in a specific tissue and not significantly excreted in urine. A mouse model was used to address these questions.

Results from the chemometric analysis on the ions produced by UPLC-TOFMS assay of control and AMK-treated mouse urines are shown in Fig. 2. The unsupervised PCA analysis revealed two clusters corresponding to the control and AMK-treated groups (Fig. 2A). The corresponding S-plot generated from OPLS-DA shows the ions that contributed to the separation of the two groups (Fig. 2B). One primary AMK metabolite (III) was observed in the urine of AMK-treated mice. Metabolite III (Fig. 2C) was detected at retention time 3.19 min, having a mass of $[\mathrm{M}+\mathrm{H}]^{+}=429.1389 \mathrm{~m} / z\left(\mathrm{C}_{18} \mathrm{H}_{26} \mathrm{~N}_{2} \mathrm{O}_{10}\right)$. The product ion at $\mathrm{m} / z 253$ was produced by loss of the glucuronic acid. The product ions at $\mathrm{m} / z, 370$ and 316 have 176 (glucuronic acid moiety) Da higher than the fragments at $\mathrm{m} / z 194$ and 140. The other product ions at $\mathrm{m} / \mathrm{z} 194,177,152,140$, and 114 underwent the same fragmentation patterns as metabolite II (Fig. 2D). Metabolite II eluted at $2.75 \mathrm{~min}$ had a protonated molecular ion at $m / z 253,16$ Da higher than that of AMK (I, Fig. 2E). MS/MS analysis of metabolite II produced product ions at $m / z 194$ (loss of $\mathrm{C}_{2} \mathrm{H}_{4} \mathrm{NO}$ ), 177 (loss of $\mathrm{C}_{2} \mathrm{H}_{4} \mathrm{NO}$ and $\mathrm{H}_{2} \mathrm{O}$ ). The product ions at $\mathrm{m} / \mathrm{z} 152$ suggest that oxidation occurred on the phenyl ring by comparison of mass fragments at $m / z 136$ from the parent compound (I, Fig. 2E). The fragment at $\mathrm{m} / \mathrm{z} 125$ further demonstrates that hydroxylation took place in the aromatic ring. The other major product ions at $m / z, 140$ and 114 were interpreted in the inlaid structural diagram (Fig. 2CE). These data suggested that AMK (I) was further metabolized in vivo to hydroxy-AMK (II, O-AMK) and glucuronide-conjugated hydroxy-AMK (III, G-O-AMK). No AMK metabolites were identified in the feces. Two other AMK metabolites have been reported in an in vitro study, including $N$-[2-(6-methoxyquinazoline-4-yl)-ethyl]acetamide and $N^{1}$ - 
acetyl-5-methoxy-3-nitrokynuramine [30]. These two metabolites were not found in this study (data not shown).

O-AMK was recapitulated in the in vitro study using HLM (Fig. 3A), and this metabolic pathway was NADPH dependent. Separate incubation of AMK with nine different human cDNA-expressed P450s revealed that CYP1A2 was the primary enzyme contributing to OAMK formation. CYP2C9, CYP2C19, and CYP2D6 were also involved in this pathway (Fig. 3B). These data suggested that formation of G-O-AMK was enzymes dependent and initiated from P450s-mediated AMK hydroxylation.

The AFMK/AMK pathway in mice was extended to O-AMK and G-O-AMK, and G-OAMK was identified as the dominant terminal AMK metabolite in vivo (Fig. 4A). In the urine of melatonin-treated mice, only trace of G-O-AMK was detected (Fig. 4B). Glucuronide-conjugated 6-hydroxy-melatonin (G-O-MEL) was the major terminal metabolite of melatonin in mice [27, 31]. Compared with the G-O-MEL pathway, G-OAMK production was minor. In addition, AFMK, AMK, and O-AMK were not observed (Fig. 4B). In human urine samples, neither O-AMK nor G-O-AMK was detected. Because of the species differences in phase II metabolism of melatonin between humans and mice [27], sulfate-conjugated O-AMK was also not detected in human urine (data not shown).

After verifying that AFMK, AMK, and their metabolites could not be detected in the urine and feces, the next step was to determine whether this was a result of the metabolites being localized in specific tissues. In serum collected $10 \mathrm{~min}$ after melatonin treatment, melatonin, G-O-MEL, and a small amount of G-OAMK were detected; nevertheless, only melatonin and G-O-MEL were observed in the serum collected at 30 min after treatment (Fig. 5A). In the livers collected at 10-min and 30-min posttreatment, melatonin and G-O-MEL were detected, but not AMK, AFMK, and G-OAMK (Fig. 5B). After identical treatment intervals, only melatonin was detected in brain (Fig. 5C) and eyes (data not shown). The tissue distribution of melatonin and its metabolites at $60 \mathrm{~min}$ were similar to that at $30 \mathrm{~min}$, but their formations were lower (data not shown). These data revealed that AFMK, AMK, and their further metabolites were not localized in brain, eye, and liver. These data suggested that AFMK/AMK pathway was minor when compared to the P450-mediated G-O-MEL pathway $[27,31]$.

In the APAP-induced oxidative stress mouse model, levels of GSH were significantly depleted in liver and a slight decrease in brain was noted (Fig. 6A/B). In addition, $2 \mathrm{hr}$ of APAP treatment had no significant effect on melatonin-metabolizing enzymes CYP1A and CYP2C (Fig. 6C). It was expected that AFMK/AMK pathway would be boosted in the APAP-induced oxidative stress mouse model. However, neither AMK nor AFMK was found in serum, liver, and brain, except for a small amount of G-O-AMK in serum (Fig. $6 \mathrm{D} / \mathrm{E} / \mathrm{F})$.

\section{Discussion}

Compelling evidence indicates that AFMK and AMK might be important melatonin metabolites and/or chemical reaction products, as several pathways including enzymatic, 
pseudo-enzymatic, and interactions with the variety of free radicals convert melatonin to AFMK and AMK [22, 32]. However, these important biogenic amines have long been neglected [30]. One reason is that there is a lack of reliable and sensitive methods for their quantification in animal specimens including fluids and tissues. Metabolomics is a rapid and systematical study of small molecule metabolites found in an organism. By using a metabolomic approach, it is possible to determine the small changes in the metabolome that take place in different groups of organisms [33]. In this study, a metabolomic approach was used to elucidate the in vivo metabolism of melatonin. Unexpectedly, AFMK/AMK was not identified in either human urine or in the urine, serum, feces, liver, brain, and eyes of mice treated with melatonin. In addition, oxidative stress induced by APAP failed to augment AFMK/AMK formation in mice. The results from the current studies indicate that the AFMK/AMK pathway plays a minor role in melatonin metabolism and degradation, especially when compared to the melatonin 6-hydroxylation pathway. The data are consistent with our previous observations in which only traces of AFMK and AMK were detected, and in some cases, they were completely undetectable in urine of mice after oral melatonin treatment $[26,27]$.

In contrast to these observations, several groups have found AFMK and AMK in animal tissues. For example, using radioactive-labeled melatonin intracisternally and intravenously injected into rats, Hirata et al. [22] claimed that the major melatonin metabolites were AFMK and AMK. By using high-performance liquid chromatography with fluorescence detection, Rozov et al. [25] confirmed that AFMK was an important melatonin metabolite in the brain of rats. In a clinical trial, high levels of AFMK have been detected in the cerebrospinal fluid in patients with meningitis. It was speculated that inflammation and oxidative stress that occurred in these patients promoted the AFMK formation from melatonin [34]. These obvious differences in the AFMK/AMK pathway are found herein and the observations of others may be as a result of the different methodologies used to detect AFMK/AMK or the use of different animal/human models. In addition, none of these previous studies compared AFMK/AMK pathway to traditional melatonin metabolic pathways, such as 6-hydroxylation and $O$-demethylation [29]. A UPLC-TOFMS-based metabolomic approach was used in this study to determine the metabolites according to retention time, accurate mass, and mass fragmentation. AFMK and AMK formations were examined in an APAP-induced oxidative stress mouse model and compared to melatonin 6hydroxylation and $O$-demethylation pathways. It is widely accepted that APAP-induced injury is mediated by a reactive metabolite, which first depletes GSH and then binds to cellular proteins and produces cellular injury. Oxidative stress was proposed as an important mechanism in APAP-induced liver injury [35, 36]. GSH is the major endogenous antioxidant produced by the cells, participating directly in the neutralization of free radicals and reactive oxygen compounds. GSH depletion was noted in this study. However, AFMK/AMK formation was not elevated.

Because of the absence of AFMK and AMK in human and mice urine, we investigated whether AMK was further metabolized or whether AFMK, AMK, and their metabolites were localized in a specific tissue. Two novel metabolites, O-AMK and G-O-AMK, were uncovered. G-O-AMK is the most abundant metabolite and has a relative abundance of over 
35 times AMK in animals treated with AMK. This study suggests that G-O-AMK is the terminal metabolite of AMK and that O-AMK serves as the intermediate metabolite between AMK and G-O-AMK. This finding extends the list of melatonin metabolites, i.e. MEL $\rightarrow$ AFMK $\rightarrow$ AMK $\rightarrow$ O-AMK $\rightarrow$ G-O-AMK. Herein, we failed to detect other AMK metabolites including $N$-[2-(6-methoxyquinazoline-4-yl)-ethyl]acetamide and $N^{1}$-acetyl-5methoxy-3-nitrokynuramine, which have been identified under in vitro conditions [30]. However, we cannot fully exclude the possibility of other AFMK/AMK metabolism pathways. Radiolabeled melatonin may help to solve the question related to the importance of kynuramines in melatonin metabolism. Among these detected AMK metabolites, none were found in humans and mouse samples analyzed in this study. O-AMK formation was enzyme dependent. The dominant enzymes that convert AMK to O-AMK are CYP1A and CYP2C isozymes, together they contribute to more than $95 \%$ of the O-AMK formed. These data raised concerns about the antioxidant functions of AMK in vivo [9], because AMK was mainly metabolized by P450s and eliminated in urine. As the final major metabolite of AFMK/AMK pathway, G-O-AMK was detected in serum after melatonin treatment, although it was extremely lower than that of G-O-MEL, a final metabolite of 6hydroxylation pathway of melatonin in mice [27, 31]. In addition, G-O-AMK and AFMK/AMK were not detected in all the tissues analyzed in this study.

In summary, the AFMK/AMK pathway was thoroughly investigated both in mice and humans using a metabolomic approach. AFMK and AMK were not identified in the urine of humans nor in the urine, feces, or tissues (including liver, brain, and eyes) of mice under the current experimental conditions. Metabolomic analysis identified two novel metabolites of AMK, O-AMK and G-O-AMK. These two newly identified metabolites were not found in the urine of humans; they were minor metabolites when compared with melatonin 6hydroxylation pathway. In addition, oxidative stress induced by APAP in the mouse model did not boost AFMK/AMK formation. These data suggested that AFMK/AMK formation is not a significant pathway in the metabolism of melatonin in mice even under oxidative stress conditions.

\section{References}

1. Reiter RJ. Pineal melatonin: cell biology of its synthesis and of its physiological interactions. Endocr Rev. 1991; 12:151-180. [PubMed: 1649044]

2. Reiter RJ. Melatonin: the chemical expression of darkness. Mol Cell Endocrinol. 1991; 79:C153C158. [PubMed: 1936532]

3. Wasdell MB, Jan JE, Bomben MM, et al. A randomized, placebo-controlled trial of controlled release melatonin treatment of delayed sleep phase syndrome and impaired sleep maintenance in children with neurodevelopmental disabilities. J Pineal Res. 2008; 44:57-64. [PubMed: 18078449]

4. Tan DX, Reiter RJ, Manchester LC, et al. Chemical and physical properties and potential mechanisms: melatonin as a broad spectrum antioxidant and free radical scavenger. Curr Top Med Chem. 2002; 2:181-197. [PubMed: 11899100]

5. Berra B, Rizzo AM. Melatonin: circadian rhythm regulator, chronobiotic, antioxidant and beyond. Clin Dermatol. 2009; 27:202-209. [PubMed: 19168001]

6. Gitto E, Pellegrino S, Gitto P, et al. Oxidative stress of the newborn in the pre- and postnatal period and the clinical utility of melatonin. J Pineal Res. 2009; 46:128-139. [PubMed: 19054296]

7. Korkmaz A, Reiter RJ, Topal T, et al. Melatonin: an established antioxidant worthy of use in clinical trials. Mol Med. 2009; 15:43-50. [PubMed: 19011689] 
8. Sofic E, Rimpapa Z, Kundurovic Z, et al. Antioxidant capacity of the neurohormone melatonin. J Neural Transm. 2005; 112:349-358. [PubMed: 15666035]

9. Ressmeyer AR, Mayo JC, Zelosko V, et al. Antioxidant properties of the melatonin metabolite N1acetyl-5-methoxykynuramine (AMK): scavenging of free radicals and prevention of protein destruction. Redox Rep. 2003; 8:205-213. [PubMed: 14599344]

10. Peschke E. Melatonin, endocrine pancreas and diabetes. J Pineal Res. 2008; 44:26-40. [PubMed: 18078445]

11. Hibaoui Y, Roulet E, Ruegg UT. Melatonin prevents oxidative stress-mediated mitochondrial permeability transition and death in skeletal muscle cells. J Pineal Res. 2009; 47:238-252. [PubMed: 19664004]

12. Pieri C, Moroni F, Marra M, et al. Melatonin is an efficient antioxidant. Arch Gerontol Geriatr. 1995; 20:159-165. [PubMed: 15374244]

13. Chegaev K, Lazzarato L, Rolando B, et al. NO-donor melatonin derivatives: synthesis and in vitro pharmacological characterization. J Pineal Res. 2007; 42:371-385. [PubMed: 17439554]

14. Schaefer M, Hardeland R. The melatonin metabolite N-acetyl-5-methoxykynuramine is a potent singlet oxygen scavenger. J Pineal Res. 2009; 46:49-52. [PubMed: 18643875]

15. Ximenes VF. Optimized synthesis of the melatonin metabolite N1-acetyl-N2-formyl-5methoxykynuramine(AFMK). J Pineal Res. 2008; 45:297-301. [PubMed: 18373552]

16. Reiter RJ, Tan DX, Manchester LC, et al. Melatonin: detoxification of oxygen and nitrogen-based toxic reactants. Adv Exp Med Biol. 2003; 527:539-548. [PubMed: 15206772]

17. Lopez A, Garcia JA, Escames G, et al. Melatonin protects the mitochondria from oxidative damage reducing oxygen consumption, membrane potential, and superoxide anion production. J Pineal Res. 2009; 46:188-198. [PubMed: 19054298]

18. Tan DX, Manchester LC, Reiter RJ, et al. Significance of melatonin in antioxidative defense system: reactions and products. Biol Signals Recept. 2000; 9:137-159. [PubMed: 10899700]

19. Tan DX, Manchester LC, Reiter RJ, et al. Cyclic 3-hydroxymelatonin: a melatonin metabolite generated as a result of hydroxyl radical scavenging. Biol Signals Recept. 1999; 8:70-74. [PubMed: 10085465]

20. Tan DX, Manchester LC, Reiter RJ, et al. Melatonin directly scavenges hydrogen peroxide: a potentially new metabolic pathway of melatonin biotransformation. Free Radic Biol Med. 2000; 29:1177-1185. [PubMed: 11121726]

21. Tan DX, Manchester LC, Burkhardt S, et al. N1-acetyl-N2-formyl-5-methoxykynuramine, a biogenic amine and melatonin metabolite, functions as a potent antioxidant. FASEB J. 2001; 15:2294-2296. [PubMed: 11511530]

22. Hirata F, Hayaishi O, Tokuyama T, et al. In vitro and in vivo formation of two new metabolites of melatonin. J Biol Chem. 1974; 249:1311-1313. [PubMed: 4814344]

23. Ferry G, Ubeaud C, Lambert PH, et al. Molecular evidence that melatonin is enzymatically oxidized in a different manner than tryptophan: investigations with both indoleamine 2,3dioxygenase and myeloperoxidase. Biochem J. 2005; 388:205-215. [PubMed: 15636586]

24. Hardeland R, Reiter RJ, Poeggeler B, et al. The significance of the metabolism of the neurohormone melatonin: antioxidative protection and formation of bioactive substances. Neurosci Biobehav Rev. 1993; 17:347-357. [PubMed: 8272286]

25. Rozov SV, Filatova EV, Orlov AA, et al. N1-acetyl-N2-formyl-5-methoxykynuramine is a product of melatonin oxidation in rats. J Pineal Res. 2003; 35:245-250. [PubMed: 14521629]

26. Ma X, Idle JR, Krausz KW, et al. Urinary metabolites and antioxidant products of exogenous melatonin in the mouse. J Pineal Res. 2006; 40:343-349. [PubMed: 16635022]

27. Ma X, Chen C, Krausz KW, et al. A metabolomic perspective of melatonin metabolism in the mouse. Endocrinology. 2008; 149:1869-1879. [PubMed: 18187545]

28. Ma X, Idle JR, Krausz KW, et al. Metabolism of melatonin by human cytochromes p450. Drug Metab Dispos. 2005; 33:489-494. [PubMed: 15616152]

29. Young IM, Leone RM, Francis P, et al. Melatonin is metabolized to N-acetyl serotonin and 6hydroxymelatonin in man. J Clin Endocrinol Metab. 1985; 60:114-119. [PubMed: 3964785] 
30. Hardeland R, Tan DX, Reiter RJ. Kynuramines, metabolites of melatonin and other indoles: the resurrection of an almost forgotten class of biogenic amines. J Pineal Res. 2009; 47:109-126. [PubMed: 19573038]

31. Kennaway DJ, Voultsios A, Varcoe TJ, et al. Melatonin in mice: rhythms, response to light, adrenergic stimulation, and metabolism. Am J Physiol Regul Integr Comp Physiol. 2002; 282:R358-R365. [PubMed: 11792644]

32. Tan DX, Manchester LC, Terron MP, et al. One molecule, many derivatives: a never-ending interaction of melatonin with reactive oxygen and nitrogen species? J Pineal Res. 2007; 42:28-42. [PubMed: 17198536]

33. Chen C, Gonzalez FJ, Idle JR. LC-MS-based metabolomics in drug metabolism. Drug Metab Rev. 2007; 39:581-597. [PubMed: 17786640]

34. Silva SO, Ximenes VF, Livramento JA, et al. High concentrations of the melatonin metabolite, N1acetyl-N2-formyl-5-methoxykynuramine, in cerebrospinal fluid of patients with meningitis: a possible immunomodulatory mechanism. J Pineal Res. 2005; 39:302-306. [PubMed: 16150112]

35. James LP, Mayeux PR, Hinson JA. Acetaminophen-induced hepatotoxicity. Drug Metab Dispos. 2003; 31:1499-1506. [PubMed: 14625346]

36. Jaeschke H, Knight TR, Bajt ML. The role of oxidant stress and reactive nitrogen species in acetaminophen hepatotoxicity. Toxicol Lett. 2003; 144:279-288. [PubMed: 12927346] 

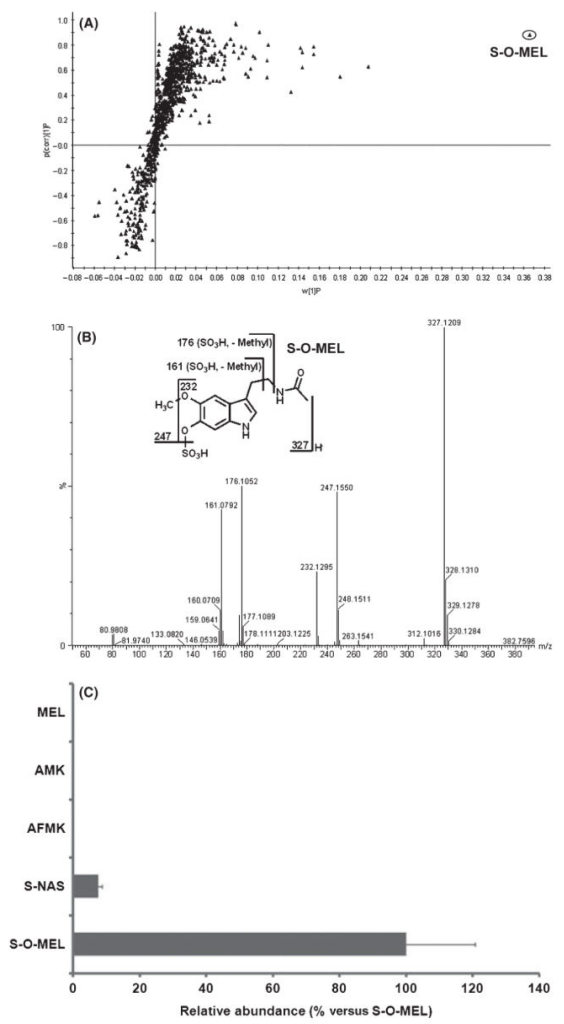

Fig. 1.

Metabolomic analysis of urines from humans pretreated with melatonin. Before and after the treatment of $10 \mathrm{mg}$ of melatonin (po), 12-hr urines were collected from healthy volunteers (n =4). (A) Loadings S-plot generated by OPLS analysis. The $X$-axis is a measure of the relative abundance of the ions, and the $Y$-axis is a measure of the correlation of each ion to the model. S-O-MEL stands for sulfated 6-hydroxymelatonin. (B) MS/MS structural elucidation of S-O-MEL $\left(\mathrm{m} / z 3^{-}\right)$. MS/MS fragmentation was conducted with collision energy ramping from 10 to $40 \mathrm{eV}$. Major product ions from fragmentation were interpreted in the inlaid structural diagrams. (C) Relative quantifications of melatonin and its metabolites S-O-MEL, S-NAS, $N^{1}$-acetyl- $N^{2}$-formyl-5-methoxykynuramine and $N^{1}$ acetyl-5-methoxykynuramine. S-NAS stands for sulfated $N$-acetylserotonin. The data were expressed as mean \pm S.E.M. The abundance of major metabolite S-O-MEL was set as $100 \%$. 

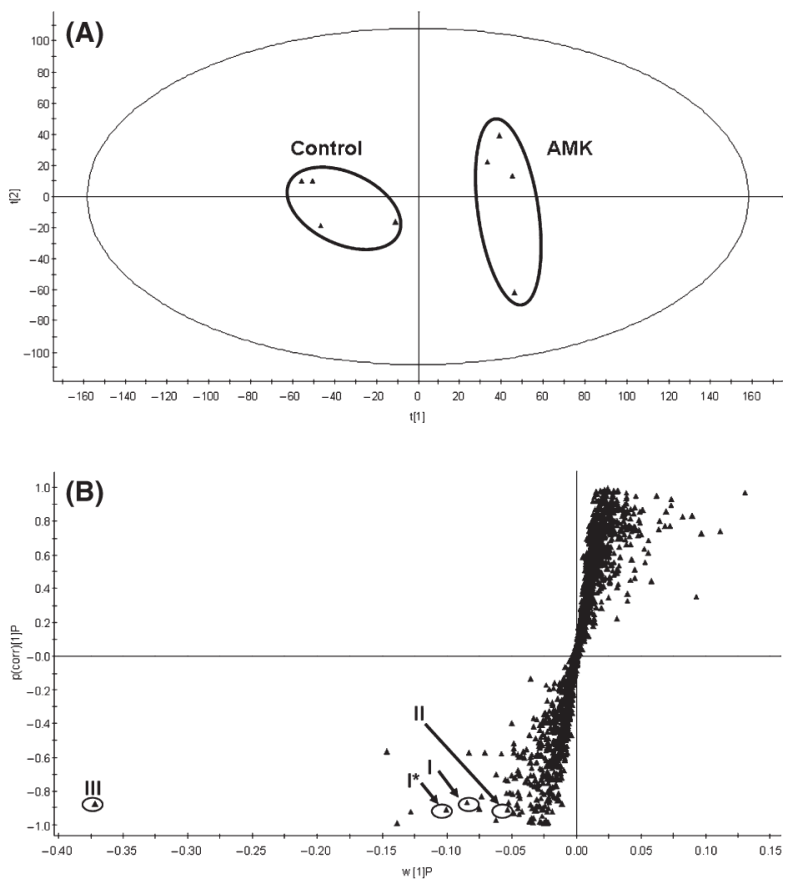

Fig. 2.
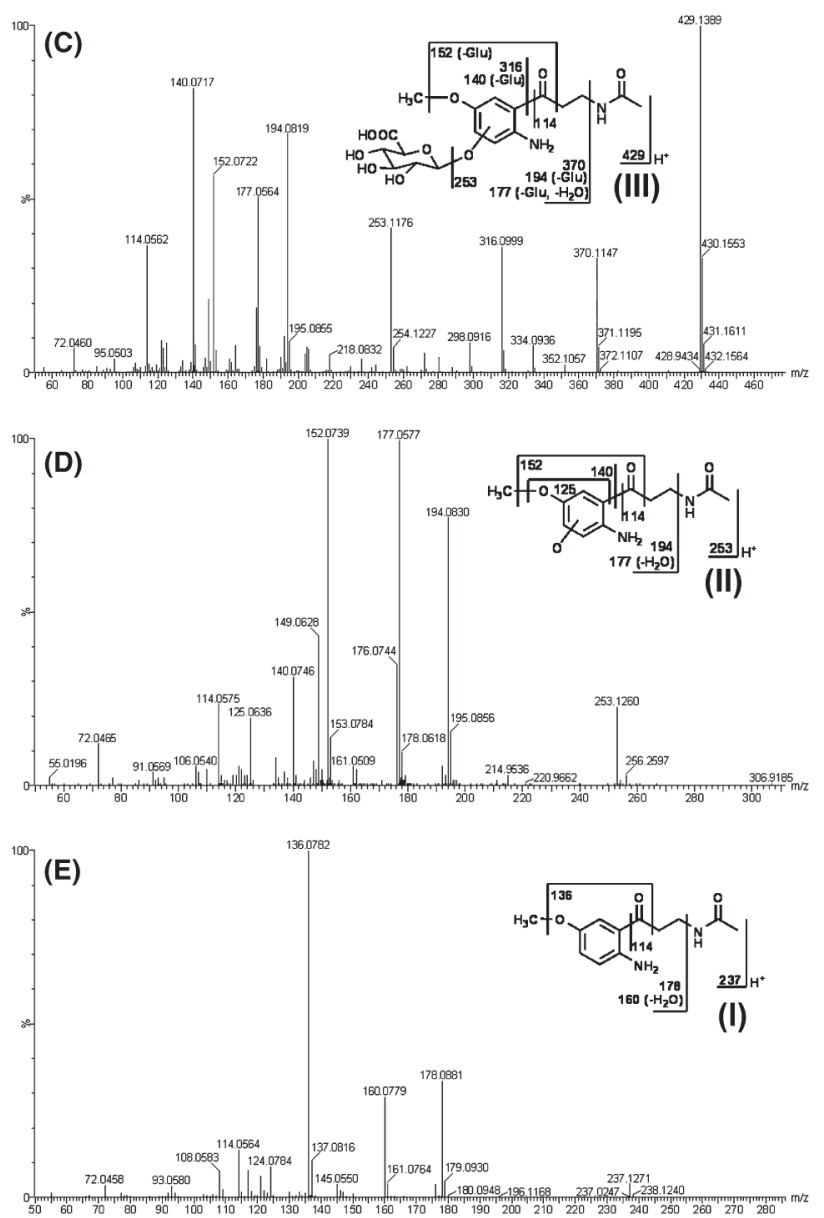

Metabolomic analysis of control and $N^{1}$-acetyl-5-methoxy-kynuramine (AMK)-treated mouse urines. WT mice $(\mathrm{n}=4)$ were treated with $10 \mathrm{mg} / \mathrm{kg}$ AMK $(\mathrm{po})$, and 18-hr urines were collected. (A) Separation of control and AMK-treated mouse urine samples in a PCA scores plot. The $t(1)$ and $t(2)$ values represent the scores of each sample in principal components 1 and 2, respectively. (B) Loadings S-plot generated by OPLS analysis. The $X$ axis is a measure of the relative abundance of the ions, and the $Y$-axis is a measure of the correlation of each ion to the model. This loadings plot represents the relationship between variables (ions) and observation groups (control and AMK treated) with regard to the first and second components present in A. AMK (I) and its metabolites (II, III) are labeled. *I stands for sodium adduct of I. (C) MS/MS fragmentation of glucuronide-conjugated hydroxy-AMK (G-O-AMK, $m / z 429^{+}$). (D) MS/MS fragmentation of hydroxy-AMK (OAMK, $\left.m / z 253^{+}\right)$. (E) MS/MS fragmentation of AMK $\left(\mathrm{m} / z 237^{+}\right)$. MS/MS fragmentation was conducted with collision energy ramping from 10 to $40 \mathrm{eV}$. Major product ions from fragmentation were interpreted in the inlaid structural diagrams. 

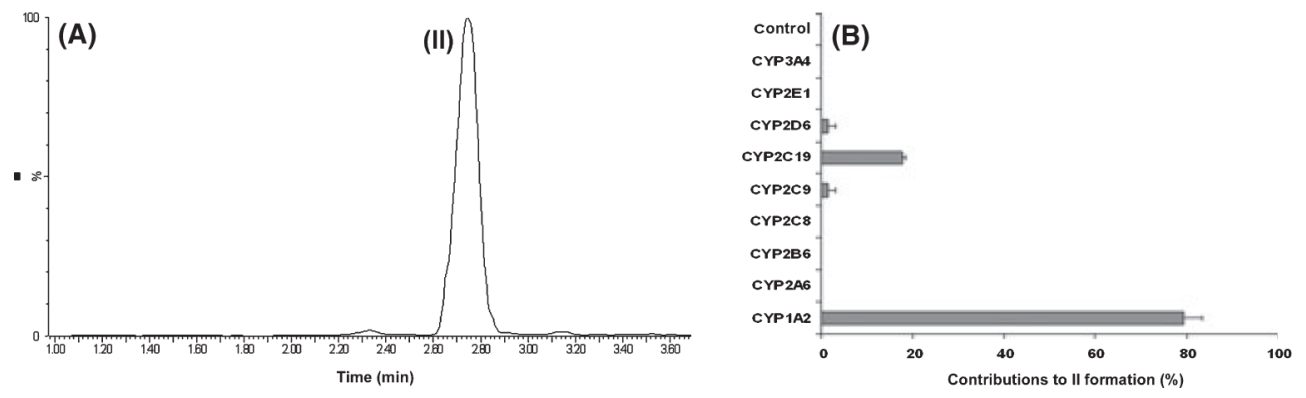

Fig. 3.

$N^{1}$-acetyl-5-methoxy-kynuramine (AMK) metabolism in vitro. The incubations were conducted in $1 \times$ phosphate-buffered saline (PBS, pH 7.4), containing $50 \mu \mathrm{m} \mathrm{AMK,} 0.1 \mathrm{mg}$ human liver microsomes (HLM), or 2 pmol of each cDNA-expressed human P450 enzyme in final volume of $200 \mu \mathrm{L}$. The reactions were initiated by the addition of $10 \mu \mathrm{L}$ of NADPH $(20 \mathrm{~mm})$ and continued for $30 \mathrm{~min}$ with shaking. (A) Representative chromatogram of AMK metabolite (II, $m / z, 253^{+}$) detected in the incubation with HLM. (B) Relative rate of AMK hydroxylation (II, $\mathrm{m} / \mathrm{z} 253^{+}$) by 9 cDNA-expressed human P450s and control insect microsomes. The data were expressed as mean \pm S.E.M. The overall contribution to AMK hydroxylation (II, $\mathrm{m} / \mathrm{z} 253^{+}$) was set as $100 \%$. 

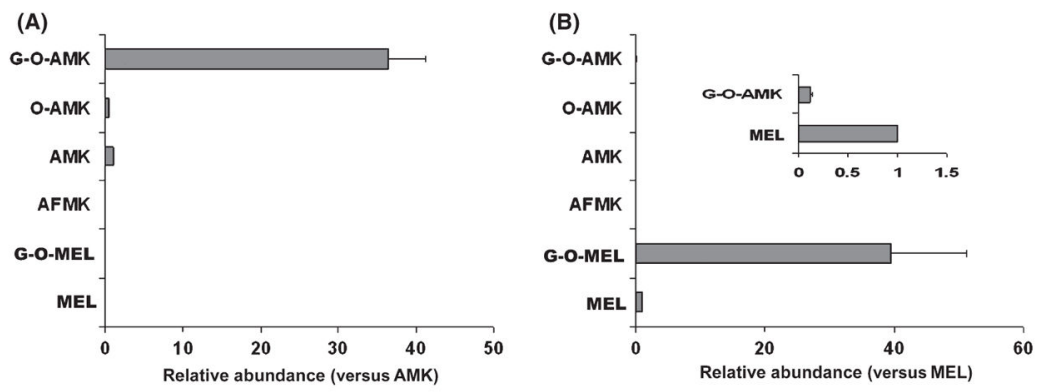

Fig. 4.

Relative quantification of major $N^{1}$-acetyl-5-methoxy-kynuramine (AMK) metabolites and melatonin metabolites in mouse urine. WT mice $(\mathrm{n}=4)$ were treated with $10 \mathrm{mg} / \mathrm{kg}$ AMK or melatonin (po). Urine samples were collected $18 \mathrm{hr}$ after treatment and analyzed by UPLCTOFMS. Screening and identification of major metabolites were performed by using MarkerLynx software based on accurate mass measurement. (A) Relative quantification of AMK metabolites. The abundance of each metabolite was represented as a ratio of peak area versus that of AMK (mean \pm S.E.M.). (B) Relative quantification of melatonin metabolites. The abundance of each metabolite was represented as a ratio of peak area versus that of melatonin (mean \pm S.E.M.). UPLC, ultra-performance liquid chromatography; TOFMS, time of flight mass spectrometry. 

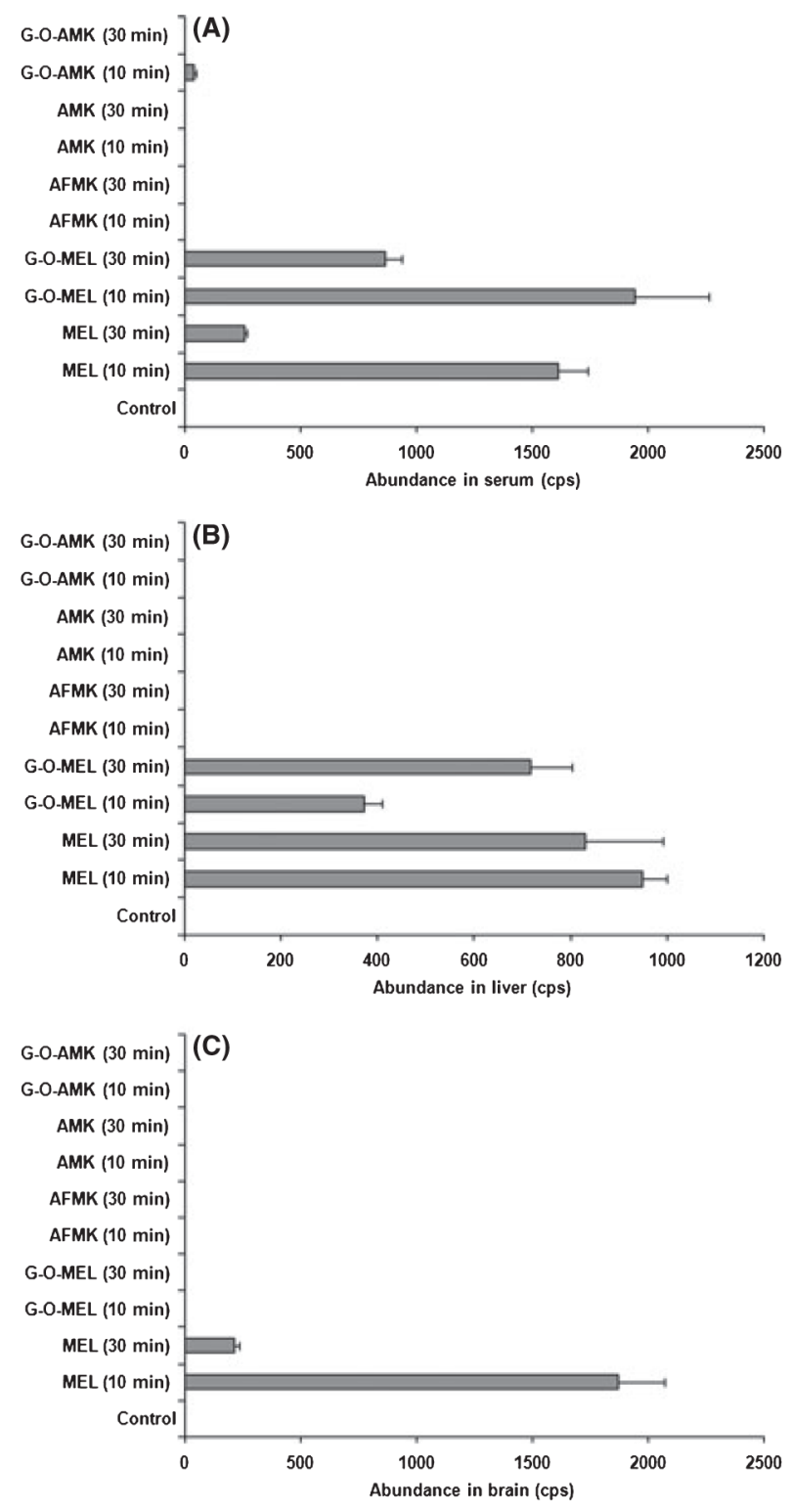

Fig. 5.

Tissue distribution of melatonin and its metabolites in mice. Blood and tissue samples from mice were collected at 10 and $30 \mathrm{~min}$ after melatonin treatment $(10 \mathrm{mg} / \mathrm{kg}$, po). Melatonin and its metabolites were analyzed by UPLC-TOFMS. The abundance (cps) of each melatonin metabolite was expressed as mean \pm S.E.M. $(n=4)$. (A) Melatonin and its metabolites in serum. (B) Melatonin and its metabolites in liver homogenate. (C) Melatonin and its metabolites in brain homogenate. UPLC, ultra-performance liquid chromatography; TOFMS, time of flight mass spectrometry. 
(A)
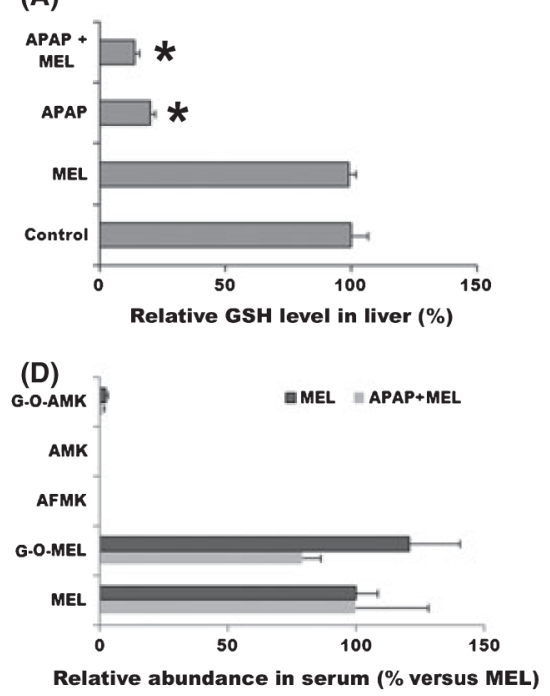

(B)
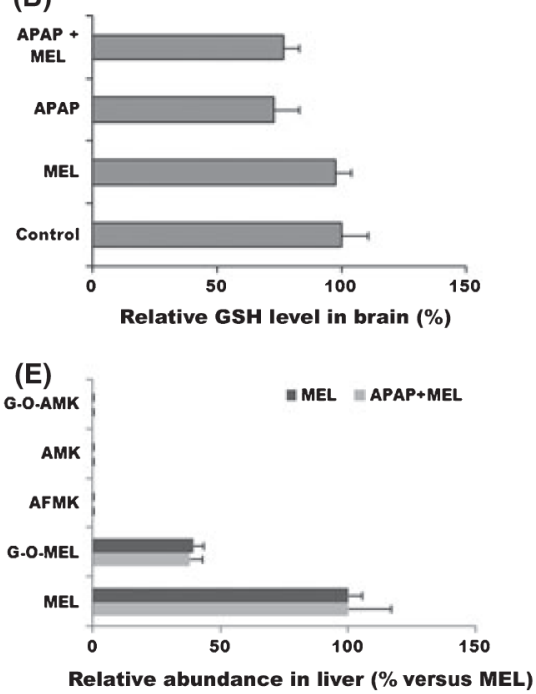

(C)
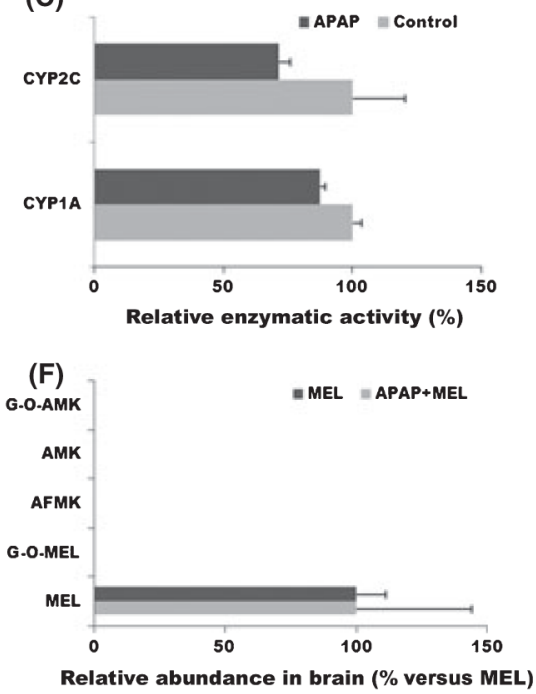

Fig. 6.

Melatonin metabolism and distribution in APAP-induced oxidative stress mouse model.

APAP (100 mg/kg, ip) was administrated $2 \mathrm{hr}$ before melatonin treatment (10 mg/kg, po).

Blood and tissue samples from mice were collected at $10 \mathrm{~min}$ after melatonin treatment. Glutathione (GSH), melatonin, and melatonin metabolites were analyzed by UPLC-TOFMS. (A) Effect of APAP treatment (2 hr) on GSH level in liver homogenate. (B) Effect of APAP treatment $(2 \mathrm{hr}$ ) on GSH level in brain homogenate. The GSH level in control group was set as $100 \%$. (C) Effect of APAP treatment (2 hr) on hepatic CYP1A and 2C activities. Enzyme activity in control group was set as $100 \%$. (D) Relative abundance of melatonin and its metabolites in serum. (E) Relative abundance of melatonin and its metabolites in liver homogenate. $(\mathrm{F})$ Relative abundance of melatonin and its metabolites in brain homogenate. The melatonin level in each group was set as $100 \%$. All data were expressed as mean \pm S.E.M. $(n=4)$. UPLC, ultra-performance liquid chromatography; TOFMS, time of flight mass spectrometry. 